\title{
Detecting System Design of Bridge Cables
}

\author{
Li Jun*
}

School of Civil Engineering and Architecture, Changsha University of Science and Technology, Changsha, 410114, China

\begin{abstract}
In this paper, a new detecting system of bridge cables is designed with a snake-shaped robot. It can solve the problems such as inefficiency and danger of aloft work in the bridge detecting by workers. The detecting system of the bridge cables includes: a snake-shaped robot; a multiple and monocular vision detection module; a nondestructive magnetic leakage detecting module; a magnetostrictive sensing module. The multiple and monocular vision detection module can detect the surface of the bridge cables. The nondestructive magnetic leakage detecting module can detect the inner part of the cables by circumferential multiple loop axial magnetization with the permanent magnets. The magnetostrictive sensing module can detect the cable fatigue and erosion. The detecting system of the bridge cables may adapt to different circumstances and complete space spiral motion, realizing the full range of the detection and increasing the detection efficiency .
\end{abstract}

Keywords: Magnetostrictive sensing module, Multiple and monocular vision detection module, Nondestructive magnetic leakage detecting module, Snake-shaped robot.

\section{INTRODUCTION}

Modern bridges, suspension bridges and cable-stayed bridges are widely used. Bridge ropes have a great influence on the working state of bridges; their service life is one major issue of concern. In the rope due to long-term exposure to natural environment, erosion inevitably is caused by the wind, sun, rain and the pollution of the environment, due to which the polyethylene (PE) sheath hardens and cracks, causing the steel wire bundle sheath corrosion. In addition, the vibration of bridge and cable stress also accelerates the cable wear and aging, producing the cable local wire break. Broken wire defect is the main factor influencing the cablestayed bridge safety and service life, and is likely to lead to catastrophic failure on the whole structure. Therefore, with the increase of traffic load and the bridge service life growth, health detection on the rope and maintenance is extremely important.

Nowadays, there are two basic methods to detect the bridges home and abroad: One is to detect the small-sized cable-stayed bridges by hydraulic or electric lifting platform, which is limited in working scopes; The other one is to detect the bridges by the worker who is in the hanging basket being dragged by the steel wire to the targeted spot installed previously on the top of the tower. The latter one is the widely used method for the detection of the suspension and cablestayed bridges, but it has many defects, for example, the manual operation may lead to inefficiency. Moreover, the aloft work is very dangerous for the workers. In short, the above two methods for detection can easily be affected by

\footnotetext{
*Address correspondence to this author at the School of Civil Engineering and Architecture, Changsha University of Science and Technology, Changsha, 410114, China; E-mail: Ljsbm523@126.com
}

the weather. In order to solve the main problems in bridge inspection, a snake like robot bridge rope detection system is designed. The system can adapt to the environment, complete space spiral motion, and achieve nondestructive detection of surface and internal injury of ropes $[1,2]$.

\section{THE STRUCTURAL COMPOSITION AND OPER- ATION PRINCIPLES OF THE DETECING SYSTEM OF BRIDGE CABLES}

This detecting system of bridge cables includes: a snakeshaped robot; a multiple and monocular vision detection module; a nondestructive magnetic leakage detecting module; a magnetostrictive sensing module. Its structural composition is shown in Fig. (1) [3].

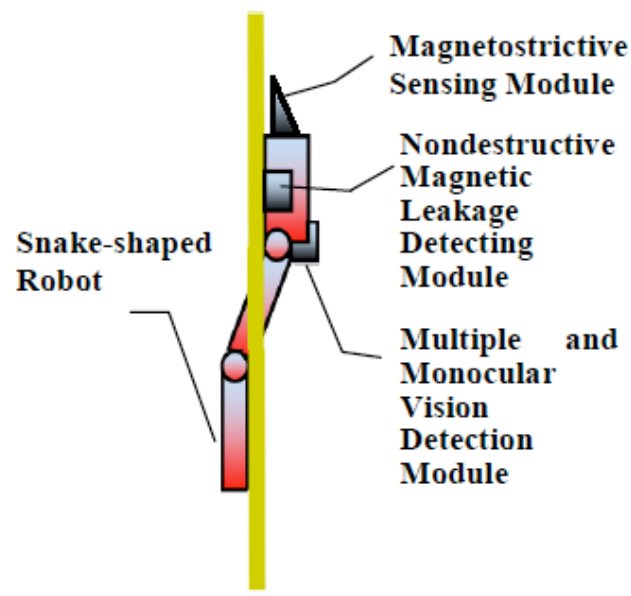

Fig. (1). The structural composition of the bridge cable detecting system. 


\subsection{The Snake-Shaped Robot}

Compared with the wheel or legged robots, the snakeshaped robots have many advantages, such as better stability, better adaptability, higher efficiency etc. Snake like robot in the bridge rope detection system based on friction plate motion decomposition synthesis transmission mechanism, uses less driven design, makes the structure more compact, and helps to reduce the body weight, reducing energy consumption $[4,5]$.

The friction disk drive is a mechanical transmission to transfer movement and power by the friction generated by the direct contact of the two disks. Its features are: the structure is simple, can change speed and direction in operation. Especially, under certain conditions, friction drive transmission ratio can change. Compared with the gear transmission, it is more flexible, and therefore more able to meet the requirements of flexibility of the snake-like robot. The relative displacement variable friction wheel transmission mechanism is shown in Fig. (2).

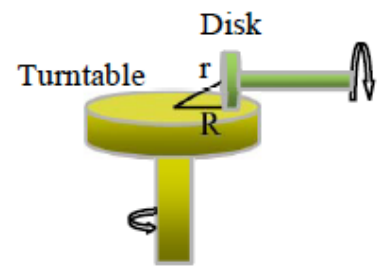

Fig. (2). Friction wheel transmission mechanism.

In the transmission mechanism as shown in Fig. (3), when the disk rotates, the effect of rolling friction drives the turntable to rotate through the rotation of the disk. On the other hand, when the turntable rotates, the rolling friction drives the disk to rotate. So the friction wheel transmission mechanism, regards the disk as well as the turn table as active movements. When the disc and the turntable are fixed, according to the principle of friction drive, the transmission ratio between them is:

$\mathrm{i}=\dot{\alpha} / \dot{\beta}=\mathrm{r} / \mathrm{R}$

Among which: $\alpha$ is the angular velocity of turntable; $\beta$ is the angular velocity of the disc; $r$ is the disc radius; $R$ is the distance of the center of the turntable to the point contact with the disk.

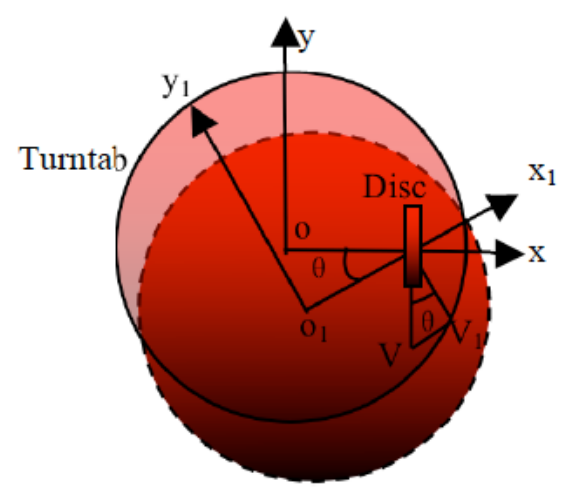

Fig. (3). The analysis of the friction disk drive mechanism.
When the turntable is turned at a relative angle $\theta$ as shown in Fig. (3), the disc line speed of the contact midpoint of the disk and the turntable is:

$\vec{V}=\gamma \dot{\beta}$

The turntable line speed of the contact midpoint of the disk and the turntable is:

$\overrightarrow{\mathrm{V}_{1}}=\mathrm{R} \dot{\beta}$

Because

$\overrightarrow{V_{1}}=\vec{V} \cos \theta$

So by formula (2) to (4) we get:

$\mathrm{i}_{\theta}=\dot{\alpha} / \dot{\beta}=\mathrm{r} \cos \theta / \mathrm{R}$

Analysis above shows that the transmission ratio is variable of the relative displacement variable friction wheel transmission mechanism. It depends on the relative configuration of the disk and the turntable.

\subsection{The Multiple and Monocular Vision Detection Module}

The multiple and monocular vision detection is an image processing technique to obtain the target information by many CCD images of different angles, obtaining the same target from different angles, and conduct matching, segmentation, subtraction treatment on multiple images. The obtained image quality is an important factor affecting the visual inspection technology [6].

In the detecting system of bridge cables, the multiple and monocular vision detection module is located on the joint part of the snake-shaped robot, which is used to detect the surface of the bridge cables. Three cameras are installed on the multiple and monocular vision detection module. Its structure is shown in Fig. (4). Among these, the light emitted by LED becomes parallel light through the optical system. The parallel light is detected on the surface of the object, and after reflection, it respectively enters into the 3 CCD. For sensor 3, the incident light angle is equal to the exit angle, which induces the bright field illumination. However, image information exhibits a deviation from round into oval shape. If the work piece diameter is $D$, parallel optical axis and the horizontal direction angle is $\alpha$, the image acquired by sensor 3 is oval, the long axis is $D / \cos \alpha$, and short axis is $d \cos \alpha$. Sensor 2 is just above the detected object, which induces the dark field illumination. It shows the actual information of the objects to be detected. Sensor 1 and the light source system work together to let light enter the lens after the reflection of the surface of object and prism, and develops dark field illumination. The image information shows a deviation from round into oval shaped too, the long axis being $D / \cos \alpha$, and short axis as $d \cos \alpha$. In the course of the experiment, $3 \mathrm{CCD}$ sensors and the position of the light source can be adjusted, the angle of incidence and the angle of reflection also available in between $0^{\circ}$ to $90^{\circ}$. Thus imaging quality can be controlled by controlling the light amount having access to $\mathrm{CCD}$, but the precondition is to ensure the outgoing angle equal to the angle of incidence. 


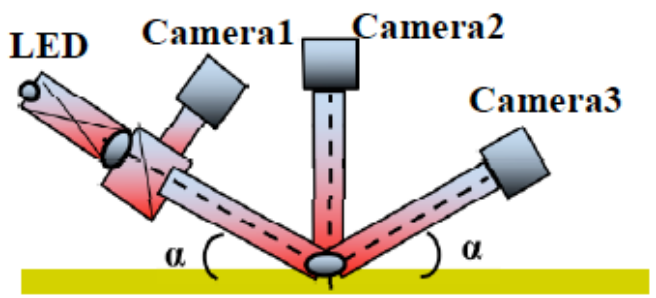

Fig. (4). The structure of the multiple and monocular vision detection module.

By using the multiple and monocular vision detection module, the textures, gradients and colors of the images can be collected and identified through template matching as well as categorized with the vector machine. And then the calculation of the image matching can be adopted when detecting the same spot of the cable surface from various perspectives. In order to improve the efficiency of the calculation, the non-ergodic image matching method can be adopted, that is, the strategy of physical stratification and logic delamination are combined during the process of image matching. Physical stratification refers to image decomposition in the form of pyramids, while logical delamination refers to rough matching preceding precise matching. Physical stratification deposes the images on the basis of the wavelet transformation. Logical delamination is achieved by the sequential similarity detection algorithm and the mean absolute difference algorithm based on the improved genetic algorithm. Then, with the combination of the physical stratification and logical delamination, the fast image matching is completed so as to identify and locate the defects of the cables.

\subsection{The Nondestructive Magnetic Leakage Detecting Module}

The materials of bridge cables possess the characteristic of high magnetoconductivity. Magnetic leakage detection is a method of finding defects by testing the changes of magnetic field on the surface of components. The principles of magnetic leakage detection can be explained by Fig. (5) [7].

The nondestructive magnetic leakage detecting module inside the snake-shaped robot is used to detect the inner part of the cables by circumferential multiple loop axial magnetization with the permanent magnets. When the to-bemeasured ferrimagnetic materials are magnetized by the magnetic saturation reactor, the magnetic induction line is bound in the materials if the materials are continuous and homogeneous. The flux is parallel with the surface of the material. With no magnetic induction line penetrating from the surface, there is no magnetic field on the measured surface. When there are defects of cutting magnetic lines in the materials, the defects on the surface of the materials or the changes in the state of the organization change the magnetic permeability. Because of the slim magnetic permeability and the great magnetic reluctance, the flux of the magnetic circuit distorts. The magnetic induction line changes its path, except a portion of flux penetrates the defects directly or bypasses the defects from the inside of the materials. Other portions of the flux leave the surface of the materials, by- pass the defects and go back into the materials through the air, resulting in a leakage of magnetic field. Thus, the distribution and size of the leakage magnetic field are detected by the magnetic sensitive sensor so as to achieve the nondestructive detection.

The rope defects can be divided into two types: one is the local defect type (Localized Fault, referred to as LF), that refers to the local position damage generated on a steel wire rope, including internal and external wire breakage, rust spots, local shape abnormality; another, the loss of metallic cross-sectional area type (Loss of Metallic Cross-sectional Area, abbreviated LMA), referring to the metallic crosssectional area which decreases damage on the cross section, including abrasion, corrosion and wire diameter shrinkage etc. $[8,9]$.

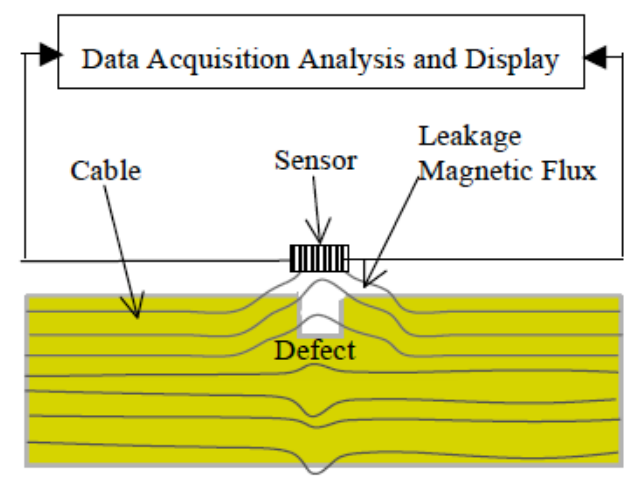

Fig. (5). The principles of magnetic leakage detecting.

The nondestructive magnetic leakage detecting module can accurately judge the type and location of defects, with the detection sensitivity being very high, even small defects causing signal fluctuation. To determine the nature of defect, it should be based on the LMA and the LF two standards, and should combine with the testing personnel having longterm accumulated experience [10].

\subsection{The Magnetostrictive Sensing Module}

Iron magnet is magnetized in a magnetic field changing the size which produces the magnetostrictive strain, resulting in the ferromagnetic body excited stress wave. The stress wave is actually a guided wave structure and is also a kind of elastic wave. On the contrary, a ferromagnetic material under action of the elastic wave produces the inverse magnetostrictive effect caused by a change in permeability or reluctance and which causes the change in magnetic induction intensity of ferromagnet. According to Faraday's law of electromagnetic induction, magnetic induction intensity change is bound to cause changes in the receiving coil voltage, so as to register the electrical measurement on the change of mechanical quantity elastic force. Acoustic encounter discontinuities that acoustic impedance is not at the same time will occur reflection, refraction and mode conversion phenomenon, therefore the acoustic echo signal in medium will contain a continuous medium with rich information, through the analysis of echo signal characteristics can be achieved for the existence of the acoustic impedance of different media such as corrosion, crack, damage judgment structure, after processing the received signal can be 
further on the types of defects, the size of the recognition, so as to realize the assessment of the structural health condition [11]. (AUTHOR: The highlighted is vague and must be rephrased for clarity)

Nondestructive testing device mainly comprises of a receiving sensor and magnetic sensor, with instrument motivation telescopic rope ultrasonic guided wave based on magnetostrictive effect; (AUTHOR: Review the highlighted correction) the device is shown in Fig. (6) $[12,13]$.

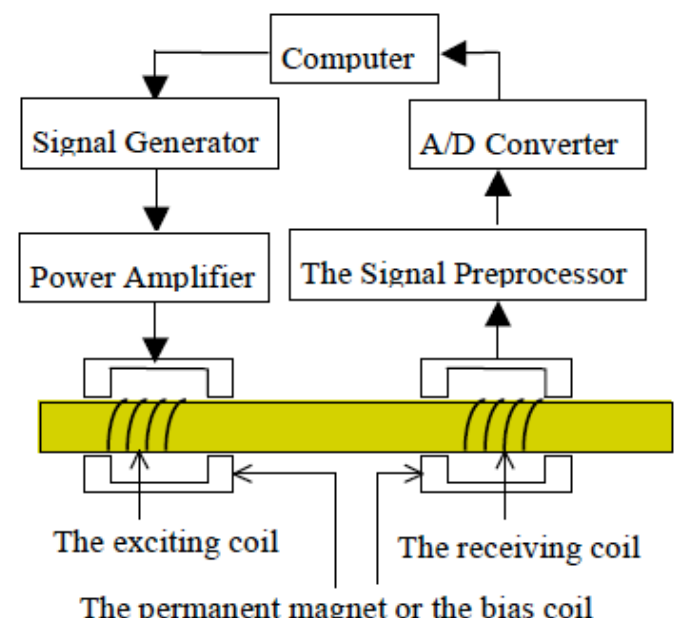

Fig. (6). The magnetostrictive guided wave rope crack detection principle.

Among the parts, the incentive sensor consists of a solenoid coil forming the bias magnetic field or permanent magnet excitation field, with the formation of the excitation coil and part of steel rope; receiving sensor consists of a solenoid coil forming the bias magnetic field or permanent magnet, along with the receiving coil and part of steel rope. The PC machine controls the signal excitation and receiving and communicates with the signal generator. The signal generator generates the excitation signal. The excitation signal is amplified by the power and loaded on the bias coil and the excitation coil [14]. The bias coil and excitation coil coaxially around the pipeline, excite magnetic fields along the rope axial and inspire the longitudinal guided waves. Guide waves in the receiving sensor are translated into electrical signals because of the inverse magnetostrictive effect, and are inputted into the A/D converter module after the disposition of the signal preprocessing module. The preprocessed signals are sent to the computer for later processing. At last, the test results are expressed in the form of images and text $[15,16]$.

The magnetostrictive sensing module, located on top of the snake-shaped robot, is used to detect the cable fatigue and erosion. The sensor is installed in the module to transmit the low-frequency guided wave signal and detect the reflected electromagnetic guided wave, with its frequency of no more than $200 \mathrm{kHz}$.

\section{CONCLUSION}

The detecting system of the bridge cables may adapt to different circumstances and complete space spiral motion through the snake-shaped robot design. With its high degree of intelligence and strong controllability, this detecting system can register the full range of the detection on both the outside and the inside and can also detect the corrosion damage of the cables by installing the multiple and monocular vision detection module as well as nondestructive magnetic leakage detecting module. This detecting system may achieve the obstacle-navigation by implementing the gait transition in detection, which may increase the detection efficiency by reducing the times of detection.

\section{CONFLICT OF INTEREST}

The author confirms that this article content has no conflict of interest.

\section{ACKNOWLEDGEMENTS}

The project was supported by Open Fund of Hunan Province University Key Laboratory of Bridge Engineering (Changsha University of Science \& Technology: 14KD07).

\section{REFERENCES}

[1] M. Mori, and S. Hirose, "Three-dimentional Serpentine Motion and Lateral Rolling by Active Cord Mechanism ACM R3," In: Proceeding IEEE Intetnational on Intelligent Robots and Systems, Swizerland, 2002, pp. 829-83.

[2] Y. Wang, Y. Kang, and X. Wu, "Magnetostrictive effect and its apphcation to NDT" , Journal of Huazhong University of Science and Technology : Nature Science Edition, vol. 33, no. 1, pp. 75-77, 2005.

[3] D. Tang, S. Huang, and W. Chen, "Study on the magnetic excitation circuit for measuring the cable tension based on magnetoelastic effect in steel cable", Chinese Journal of Scientific Instrument,vol. 27, no. 12, pp. 1695-1699, 2006.

[4] Z. Liu, Y. Zhang, and H. Zhang, "Experimental research on excitation and reception of longitudinal guided wave modes in steel strands based on magnetostrictive effect," Journal of Mechanical Engineering,vol. 46, no. 6, pp. 71-76, 2010.

[5] Q. S. Cao, D. Liu, and Y. H. He, "Nondestructive and Quantitative Evaluation of Wire Rope Based on Radial Basis Function Neural Network using Eddy Current Inspection," NDT\&E International, vol. 46, pp. 7-13, 2012.

[6] H. Henao, S. M. J. R. Fatemi, and G. A. Capolino, "Wire rope fault detection in a hoisting winch system by motor torque and current signature analysis", IEEE Transaction on Industrial Electronics, vol. 58, no. 5, pp. 1727-1736, 2011.

[7] A. Demma, P. Cwaley, and M. Lowe, "The reflection of guided waves from notches in pipes: a guide for interpreting corrosion measurements," NDT\&E International, vol. 37, pp. 167-180, 2004.

[8] T. Calkins, A. B. Flatau, and M. J. Dapino "Overview of magnetostrictive sensor technology" Journal of Intelligent Material Systems and Structures, vol. 18, no. 10, pp. 1057-1066, 2007.

[9] L. Laguerre, J. C. Aime, and M. Brissaud, "Magnetostrictive pulseecho device for non-destructive evaluation of cylindrical steel materials using longitudinal guided waves" Ultrasonics, vol. 39, no. 7, pp. 503-514, 2002.

[10] J. L. Rose, "Recent advances in guided wave NDE," In: Proceedings of the IEEE Ultrasonics Symposium, Seattle, WA, USA, Nov 07-10, 1995, Piscataway : IEEE, vol. 1, pp. 761-770, 1995.

[11] H. Takahiro, S. Won-Joon, and J. L. Rose, "Guided wave dispersion Curves for a bar with all arbitrary cross-section, a rod and rail example," Ultrasonics, vol. 4l, no. 3, pp. 175-183, 2003.

[12] H. Takahiro, K. Koichiro, Z. Sun, and J. L. Rose, "Analysis of flexural mode focusing by a semi analytical finite element meth- 
od," Journal of the Acoustical Society of America, vol. 113, no. 3, pp. 1241-1248, 2003.

[13] W. Gu, and J. X. Chu, "A transducer made up of fluxgate sensors for testing wire rope defects," IEEE Transactions on Instrumentation and Measurement, vol. 51, no. 1, pp. 120-124, 2002.

[14] V. Reimund, M. Blome, and M. Pelkner, "Fast defect parameter estimation based on magnetic flux leakage measurements with
GMR sensors," International Journal of Applied Electromagnetics and Mechanics, vol. 37, pp. 199-205, 2011.

[15] W. Sharatchandra, B. P. C. Rao, and S. Thirunavukkarasu, "Flexible GMR sensor array for magnetic flux leakage testing of steel track ropes" Journal of Sensors, vol. 2012, pp. 1-6, 2012.

[16] C. Y. Xiao, and Y. H. Zhang, "A Method of Magnetic Scanning Imaging for Detecting Defects in Ferromagnetic Materials," Measurement Science and Technology, vol. 22, pp. 1-7, 2011.

Received: February 03, 2015

Revised: April 03, 2015

Accepted: May 25, 2015

(C) Li Jun; Licensee Bentham Open.

This is an open access article licensed under the terms of the (https://creativecommons.org/licenses/by/4.0/legalcode), which permits unrestricted, noncommercial use, distribution and reproduction in any medium, provided the work is properly cited. 